\title{
ATMOSPHERIC EFFECTS ON INTERFEROMETRIC OBSERVATION
}

\author{
Han Wen-jun \\ Beijing Observatory \\ Academia Sinica \\ Beijing, People's Republic of China
}

In recent years, the influence of atmospheric irregularities on electronic systems has been recognized. How these irregularities affect electronic systems is related to the propagation environment, the scales of the irregularity, and parameters of the equipment. The phase deviation caused by the atmosphere is important to the interferometer. However, there are some filter effects, namely antenna aperture filter, Fresnel filter, and baseline filter, which must be considered. The first two filter out the effects of small scale irregularities, and the last one filters out larger ones.

The atmospheric influence on centimeter wavelength and meter wavelength radio waves is mainly due to the troposphere and ionosphere, respectively. Concerning the excess propagation path, these two effects on observation have opposite signs. Taking the operating frequency to be about $2200 \mathrm{MHz}$ under normal conditions, these two effects cancel each other to first order. In the meter waveband, because of the larger beam of the antenna, the ground reflected wave must be considered at large zenith angle, especially in aperture synthesis systems. The phase deviation caused by this effect is more serious, since the antenna heights for each set referred to its reflecting plane are in practice not equal.

The effect of atmospheric inhomogeneities on the interferometer is caused by horizontal gradient of inhomogeneity. If the baseline is not longer than about $1 \mathrm{~km}$, the phase deviation caused by average atmosphere (quiet condition) is not larger than about $20^{\circ}$ at $2 \mathrm{GHz}$, but that caused by irregularities (disturbed condition) is serious. The irregularities of the troposphere are due to the uneven distribution of water vapor in the form of clouds, fronts, etc., and those of the ionosphere are due to travelling ionospheric disturbance (TID), spread F and sporadic E. A model of sporadic $\mathrm{E}$ is suggested, and the effect of this on meter wave aperture synthesized beam is computed. The following conclusions are drawn: the gain of the main lobe of the synthesized beam decreases about 10 percent, and a right ascension error of about 0.6 second is introduced.

M. J. Reid and J. M. Moran (eds.), The Impact of VLBI on Astrophysics and Geophysics, 545.

(c) 1988 by the IAU. 